\title{
Starvation ketoacidosis in pregnancy presenting as euglycaemic, high anion gap metabolic acidosis: A case report highlighting the significance of early recognition and prompt intervention
}

\author{
J M Chausse, ${ }^{1} \mathrm{MB}$ ChB; F Paruk, ${ }^{1} \mathrm{MB}$ ChB, PhD (Clinical Medicine), Cert Critical Care (SA), FCOG (SA); S Motilall, ${ }^{1}$ MB ChB, FCS (SA), \\ Cert Critical Care (SA), Cert Trauma (SA); P Soma-Pillay, ${ }^{2}$ FCOG, Cert Maternal and Fetal Medicine (SA), PhD; $\mathbf{S}$ Ndaba, ${ }^{2} \mathrm{MB}$ ChB \\ ${ }^{1}$ Department of Critical Care, School of Medicine, Faculty of Health Sciences, University of Pretoria and Steve Biko Academic Hospital, \\ Pretoria, South Africa \\ ${ }^{2}$ Department of Obstetrics and Gynaecology, School of Medicine, Faculty of Health Sciences, University of Pretoria and Steve Biko Academic \\ Hospital, Pretoria, South Africa
}

Corresponding author: F Paruk (fathima.paruk@up.ac.za)

\begin{abstract}
Starvation ketoacidosis (SKA) constitutes an important consideration in the pregnant patient who presents with profound metabolic acidosis. Pregnancy-related changes predispose the patient to develop SKA following relatively short periods (12 - 14 hours) of 'starvation'. Patients also typically look clinically well in relation to the significant metabolic derangements that accompany the condition. Prompt recognition and early institution of appropriate therapy is therefore extremely important in terms of optimising maternal and fetal outcome. We describe a pregnant patient with SKA who presented with profound euglycaemic ketoacidosis that resolved rapidly following the early initiation of appropriate therapy. Furthermore, appropriate therapy resulted in our patient avoiding the need for an emergency caesarean section, which is often reported in this scenario. The ensuing discussion addresses SKA in pregnancy, the unique features of our patient, and management considerations from a maternal and fetal perspective. We also discuss the various causes of ketoacidosis such as diabetic ketoacidosis (DKA), euglycaemic DKA, alcohol-induced euglycaemic ketoacidosis and SKA in pregnant patients.
\end{abstract}

S Afr Med J 2018;108(8):636-639. DOI:10.7196/SAMJ.2018.v108i8.13082

The occurrence of metabolic acidosis during pregnancy necessitates prompt evaluation and intervention taking into account the potential risks of poor maternal and/or fetal outcomes. ${ }^{[1]}$ Metabolic acidosis attributed to starvation is referred to as starvation ketoacidosis (SKA) ${ }^{[1,2]}$ It is not commonly encountered during pregnancy, and the diagnosis may often be delayed as initially patients frequently look clinically disproportionately well, despite the presence of severe metabolic acidosis. A delay in initiation of therapy may result in profound metabolic acidosis and its sequelae.

SKA is extremely rare in non-pregnant individuals. Moreover, it differs from SKA in pregnancy in that the acidosis is initially milder, and at least 10 - 14 days of starvation are required for the ketoacidosis to manifest clinically. ${ }^{[3]}$ SKA has been described as occurring in the settings of gastric banding, severe malnutrition and the Atkins diet. It has been demonstrated that in the pregnant state the risk of developing ketosis is accelerated and it can manifest after only 12 - 14 hours. ${ }^{[4,5]}$ This predisposition to the rapid development of ketosis is largely driven by a multitude of factors including the insulin resistance, enhanced lipolysis and ketogenesis that occur during pregnancy. ${ }^{[6]}$ Human placental lactogen, cortisol and glucagon, which are produced by the placenta, further exacerbate SKA.

We describe a patient with SKA in pregnancy who presented with profound euglycaemic ketoacidosis that resolved rapidly following the early initiation of appropriate therapy, resulting in a good maternal and fetal outcome. Additionally, the early recognition and initiation of appropriate therapy resulted in our patient recovering rapidly and avoiding the need for an emergency caesarean section, which is often reported in this scenario. ${ }^{[7]}$ The ensuing discussion addresses SKA in pregnancy, the unique features of our patient, and management considerations from a maternal and fetal perspective.

\section{Case report}

A 24-year-old woman, para 2 gravida 3, presented to her local primary care hospital at 36 weeks' gestation with a 2-day history of malaise and worsening lower abdominal pains. She had been receiving antenatal care at her local clinic. Her two previous pregnancies had been uncomplicated. In the index pregnancy, her antenatal history and clinical course were unremarkable. There was no previous medical or surgical history of note, including impaired glucose tolerance, alcohol use or use of chronic medications.

On presentation at the primary care hospital the patient had slight tachycardia, but the rest of her vital signs, including temperature, blood pressure and respiratory rate, were within the normal range for a pregnant woman. Her heart sounds were normal and her chest was clinically clear. The fetus was misjudged to be of 34 weeks' gestational age. She had a longitudinal lie with a cephalic presentation, and three mild contractions were palpable over a 10-minute interval. The cardiotocogram (CTG) was normal and urinalysis revealed leucocytosis with $2+$ ketones and a trace of protein. Capillary blood glucose was not recorded as glucometers were out of stock at the primary care facility. The patient was admitted with a provisional diagnosis of preterm labour precipitated by a urinary tract infection. 
Treatment included an infusion of Ringer's lactate, the initiation of azithromycin and metronidazole, and administration of a calcium channel blocker for medical tocolysis. She was discharged from the facility several hours later. The following day she returned to the same facility complaining of nausea and vomiting. She was noted to be tachypnoeic, and concerns were raised as fetal heart rate decelerations with a concomitant increase in uterine activity were noted on the CTG. She was therefore transferred to a secondary healthcare hospital.

On arrival at the secondary facility the patient was managed as being in preterm labour. She was haemodynamically stable and the CTG was normal. Urinalysis once again depicted $2+$ ketones. A Ringer's lactate infusion and ceftriaxone were initiated and she was given betamethasone for fetal lung maturation. While at the facility, her respiratory status was observed to deteriorate (progressively more tachypnoeic with laboured breathing) and she was therefore transferred to our tertiary centre (Steve Biko Academic Hospital, Pretoria, South Africa (SA)).

On admission the patient was noted to be apyrexial. She had a sinus tachycardia of $131 \mathrm{bpm}$ and was normotensive and tachypnoeic, with a respiratory rate of 40 breaths per minute with the use of accessory muscles evident. The remainder of the clinical examination was unremarkable in that her heart sounds were normal, air entry was equal bilaterally, and her abdomen was non-tender with a gravid uterus corresponding to 36 weeks' gestation. Arterial blood gas measurement revealed severe metabolic acidosis $\left(\mathrm{pH}\right.$ 7.12, $\mathrm{PaO}_{2}$ (arterial partial pressure of oxygen) $85 \mathrm{mmHg}, \mathrm{PaCO}_{2}$ (arterial partial pressure of carbon dioxide) $12.8 \mathrm{mmHg}$, base excess -24.6, bicarbonate $7.3 \mathrm{mmol} / \mathrm{L}$, lactate $2.1 \mathrm{mmol} / \mathrm{L}$, albumin $32 \mathrm{~g} / \mathrm{dL}$, anion gap (AG) $26 \mathrm{mmol} / \mathrm{L}$ and blood glucose $5.4 \mathrm{mmol} / \mathrm{L}$ on $0.4 \mathrm{FiO}_{2}$ (fraction of inspired oxygen). Urinalysis revealed ketonuria 3+ with a trace of protein. Her electrolytes, liver function tests and full blood count were within an acceptable range. No abnormalities were detected on a toxin screen and blood cultures were subsequently found to be negative. The patient was admitted to the intensive care unit (ICU), where resuscitation including mechanical ventilation was initiated. A tentative diagnosis of SKA in pregnancy was considered and the patient was initiated on an intravenous $10 \%$ dextrose solution, whereupon her metabolic parameters improved dramatically with normalisation of the $\mathrm{pH}$ within 6 hours of appropriate therapy. She was liberated from the ventilator within 14 hours. A computed tomography pulmonary angiogram excluded the possibility of a pulmonary embolus. The fetus was monitored once the patient's $\mathrm{pH}$ and physiological status improved and the CTG was reassuring during this period. She was discharged from the ICU within 60 hours after admission and went on to have an uncomplicated normal vaginal delivery a few days later, delivering a $2700 \mathrm{~g}$ male infant with good Apgar scores of 8/10 at 1 minute and $9 / 10$ at 5 minutes. No subsequent complications, including re-feeding syndrome, were observed. Mother and baby were discharged 7 days after admission to the tertiary hospital.

\section{Discussion}

\section{Metabolic acidosis in pregnancy}

Metabolic acidosis in pregnancy may be life-threatening to both the mother and the fetus. ${ }^{[1,3]}$ Its occurrence, albeit rare, is generally considered to indicate a significant underlying disease process. It is characterised by an increase in plasma acidity caused by an acid-base disturbance. The AG is useful in teasing out the aetiology. A normal AG metabolic acidosis is seen in patients with increased bicarbonate loss either from the gastrointestinal tract due to diarrhoea, pancreatic fistulas and ureteroenterostomies or through renal losses as seen in chronic renal failure as well as in type 2 renal tubular acidosis. Additional causes of a normal AG metabolic acidosis include the use of carbonic anhydrase inhibitors such as acetazolamide or chloridecontaining acids such as ammonium chloride, where the loss of bicarbonate is accompanied by a rise in chloride due to compensatory reabsorption of chloride by the kidneys as well as exogenous supplementation. Reduced renal acid clearance (types 1 and 4 renal tubular acidosis) may also result in a normal AG metabolic acidosis.

An elevated AG ( $>16 \mathrm{mmol} / \mathrm{L})$ acidosis may be attributed to hyperlactaemia (e.g. sepsis), excessive ketone production (diabetes mellitus, starvation, alcohol intake or certain inborn errors of metabolism, or with genetic predisposition), uraemia, acute fatty liver of pregnancy, excessive alcohol consumption, or ingestion of exogenous acids such as in poisoning with methanol, salicylates or ethylene glycol.

Our patient presented with a history of vomiting but no recent ingestion of alcohol or impaired glucose tolerance in the antenatal period. Taking into consideration that the lactate level was at the upper limit of the normal range, her euglycaemia, a fetus appropriate for gestational age, recent corticosteroid exposure and a high AG ketoacidosis, we considered that SKA was the most likely diagnosis.

\section{Ketoacidosis in pregnancy}

The heightened hormonal drive (cortisol, human placental lactogen and glucagon), insulin resistance, elevated resting serum ketone concentration and reduced ability to buffer hydrogen ions (chronic respiratory alkalosis and a compensatory metabolic acidosis) together with the state of accelerated starvation in pregnancy predispose the pregnant woman to rapid development of ketoacidosis compared with her non-pregnant counterpart. This is especially the case in the third trimester, and the process is rapidly exacerbated by stress, insulin resistance or short periods of starvation.

The commonest cause of ketoacidosis in pregnancy is type 1 diabetes mellitus. Diabetic ketoacidosis (DKA) is the most common cause of metabolic acidosis and is typically seen in type 1 diabetes as a result of insulin deficiency. The insulin deficiency may be attributed to poor plasma glucose monitoring, inadequate treatment or an underlying infection. Patients typically present with elevated serum glucose levels. Euglycaemic DKA (EDKA) is also a well-described entity and is discussed below.

The differential diagnosis for euglycaemic ketoacidosis in pregnancy needs to include EDKA, ketoacidosis associated with starvation, or excessive alcohol intake. The most likely cause needs to be elucidated rapidly so that appropriate management strategies can be implemented timeously.

\section{EDKA}

Typically the diagnosis of DKA can be made easily if the patient has hyperglycaemia (serum glucose $>11 \mathrm{mmol} / \mathrm{L}$ ), venous $\mathrm{pH}<7.3$ and/or bicarbonate $<15 \mathrm{mmol} / \mathrm{L}$ ). In pregnancy, however, DKA may occur even with normal or slightly elevated serum glucose levels. This is attributed to the increased risk of ketosis in pregnancy as well as the fact that the fetus preferentially utilises maternal glucose stores. Several definitions have been proposed for EDKA..$^{[8-11]}$ For practical purposes, EDKA should be considered in pregnancy in the scenario of ketoacidosis with a serum glucose level $<11.1 \mathrm{mmol} / \mathrm{L}$. The presenting clinical scenario is useful in confirming or refuting the diagnosis. In the absence of a history of impaired or abnormal glucose tolerance, features that suggest a diagnosis of EDKA include a mildly elevated blood glucose level, polydipsia, glycosuria, a large for gestational age fetus, polyhydramnios, worsening glycaemic control or increasing ketones on stopping insulin therapy. 
Our patient did not exhibit the above features of EDKA, and her serum glucose also remained normal post treatment of the SKA until discharge from the hospital. EDKA therapy essentially entails the concomitant administration of insulin and dextrose water (as one would with DKA once the serum glucose level is $<14 \mathrm{mmol} / \mathrm{L}$ ), as well as monitoring of serum glucose levels, electrolytes and fluid balance.

\begin{abstract}
Alcohol-induced euglycaemic ketoacidosis
Alcohol-related ketoacidosis occurs most commonly in the setting of type 1 diabetes, but may also predispose to significant ketoacidosis on its own. This typically occurs in the setting of excessive alcohol consumption followed by prolonged vomiting. The ketone production is mainly attributed to starvation, but acetate conversion to ketones also plays a role. Beta-hydroxybuyrate comprises the predominant ketone produced. Typically the patient may present with euglycaemia or hypoglycaemia due to nicotinamide adenine dinucleotide (NAD) consumption during the conversion of alcohol to acetate. The mainstay of treatment is intravenous administration of dextrose.
\end{abstract}

\section{SKA in pregnancy}

In a healthy non-pregnant individual it takes up to 14 days to reach the maximum severity of SKA, which would manifest with mildly elevated ketoacid levels and minimal acid-base disturbances. ${ }^{[2]}$ In pregnancy, on the other hand, apart from an accelerated early manifestation of ketoacidosis, the severity is also much more profound. This unbalanced and dramatic response to fasting can manifest with increased ketone formation and markedly increased levels of free fatty acids and $\beta$-hydroxybutyrate within 12 hours. ${ }^{[4,5]}$

In the scenario of low glucose availability, a reduced pool of pyruvate enters the citric acid cycle as a result of depletion of glycogen stores. Energy production is therefore provided by the generation of acetyl CoA from beta-oxidation of free fatty acids. This may exceed the capacity of the citric acid cycle leading to the accumulation of $\beta$-hydroxybutyrate, acetoacetate and acetone, which results in metabolic acidosis. ${ }^{[12]}$

\section{Presentation and diagnosis}

Our case bears a noticeable resemblance to other cases of SKA reported in the literature in that the patient presented in her third trimester, had a recent onset of nausea and vomiting that was of very short duration ( $<24$ hours), and was tachypnoeic and tachycardic with profound acid-base disturbances. Biochemical similarities include an acidic $\mathrm{pH}$ ranging between 7.02 and 7.31, a low bicarbonate level of $<9 \mathrm{mmol} / \mathrm{L}$ and a glucose level in the normal to low-normal range, as well as a high AG and ketonuria. While these features may also conform to the criteria for EDKA, we have explained why we considered that the patient had SKA rather than EDKA.

Several factors may have influenced the patient's clinical picture. She had experienced a relatively short period of fasting/poor nutritional intake due to the nausea and vomiting in conjunction with a relative insulin-deficient state associated with pregnancy (which is maximal in the third trimester), largely attributed to counterregulatory, placentally derived hormones that are up-regulated during periods of stress, further aggravating the metabolic derangement. Table 1 addresses the clinical approach to evaluating patients who present with severe ketoacidosis in pregnancy.

\section{Exacerbating conditions}

The administration of betamethasone at the secondary facility for fetal lung maturation could have contributed to the patient's insulin resistance and worsened the ketoacidosis. As tocolytic agents,
Table 1. Approach to ketoacidosis in pregnancy

\begin{tabular}{ll}
\hline History & Nutrition (last intake and vomiting) \\
& Headache \\
& Blurred vision \\
& Alcohol \\
& Glucose intolerance \\
Clinical assessment & Comprehensive assessment of all organ \\
& systems, including respiratory rate, \\
& blood pressure, Glasgow Coma Score, \\
& temperature, evidence of infection \\
& Urine dipstick (ketonuria, nitrites, \\
& leucocytes) \\
& Serum glucose \\
& Urea and electrolytes \\
& Full blood count \\
& Capillary ketones \\
& Inflammatory markers (C-reactive \\
& protein, procalcitonin) \\
& Arterial blood gas \\
& Anion gap \\
& Diabetic ketoacidosis \\
& Euglycaemic diabetic ketoacidosis \\
& Starvation ketoacidosis \\
Alcohol-induced euglycaemic diabetic \\
ketoacidosis \\
Gene defects \\
\end{tabular}

$\beta$-agonists have often been associated with ketoacidosis in diabetic as well as non-diabetic women as they increase lipolysis, gluconeogenesis and glycogenolysis. ${ }^{[13]}$ The administration of azithromycin and metronidazole could have further exacerbated the vomiting in our patient. It is therefore vitally important to consider the implications of seemingly innocuous agents prior to their prescription in pregnant patients.

\section{Management considerations}

A $10 \%$ dextrose solution is probably more effective than a $5 \%$ solution in patients with SKA. A recent study demonstrated the pitfalls of a $5 \%$ dextrose solution, where its use resulted in worsening rather than improvement of the ketoacidosis. ${ }^{[14]}$ It could be that the relatively low glucose content of a $5 \%$ dextrose solution does not provide an adequate amount of glucose. Furthermore, the rapid metabolism of the dextrose renders the solution hypotonic, so that it does not remain in the intravascular compartment for a reasonable duration and therefore extravasates into the surrounding tissues, which in turn worsens the acidosis. Maternal and fetal hyponatraemia is of concern when resuscitating a pregnant patient with salt-free fluids, and an infusion of normal saline for volume replacement administered alongside an infusion of $10 \%$ dextrose may avoid this complication. We used a $10 \%$ dextrose solution as well as $0.9 \%$ normal saline to address the glucose and fluid replacement requirements, respectively.

Caution is recommended when administering intravenous dextrose-containing solutions in patients with prolonged fasting, as it may be associated with complications such as Wernicke's encephalopathy and central pontine myelinolysis. In the scenario of hypoglycaemia, 50\% dextrose should be administered via a central catheter as it may cause irritation when administered peripherally.

Neonatal hyperinsulinaemia and therefore hypoglycaemia was previously thought to be of concern following the administration of dextrose to women in labour. Recent studies indicate that symptomatic 
neonatal hypoglycaemia is not a concern in this scenario. ${ }^{[15,16]}$ In the case of SKA in pregnancy the duration of fasting is relatively short, which reduces the possible risk of re-feeding syndrome; however, a high index of suspicion is needed and monitoring of blood biochemistry, including calcium, magnesium and phosphate levels, would be prudent.

\section{Therapy - fluids, sodium bicarbonate and insulin}

There is no evidence to support the use of sodium bicarbonate in SKA. ${ }^{[12]}$ Its administration is therefore controversial and the routine use of intravenous sodium bicarbonate is not recommended. The worsening of intracellular acidosis and the occurrence of hypokalaemia or cerebral oedema are concerns related to its administration. While there is no clear evidence of a reduction in morbidity or mortality, one may consider administering it in severe metabolic acidosis with a $\mathrm{pH}<6.9$.

\section{Mode of delivery}

Many case reports refer to the need for an emergency caesarean section in SKA in pregnancy ${ }^{[7,12,17]}$ This practice may be due to the belief that delivering the fetus and placenta would correct the ongoing metabolic abnormality by removal of the placentally driven counter-regulatory hormones. We would like to highlight that an emergency caesarean section in an unstable, poorly optimised, acidotic mother may in fact predispose her to complications such as excessive bleeding and further deterioration of the metabolic derangement. Burbos et al. ${ }^{[18]}$ describe a patient with features of ketoacidosis who was not taken for an emergency caesarean section and demonstrated rapid improvement of her metabolic status following intravenous administration of $10 \%$ dextrose. The emphasis should in fact be on early recognition of the condition and initiation of therapy to restore the metabolic milieu to normality. Our patient received prompt initiation of appropriate therapy, with rapid resolution of the fetal distress that was initially reported at the referral hospital (CTG at referral hospital). We initiated CTG monitoring as soon as the patient's haemodynamic and metabolic status was considered stable, which was within a few hours after ICU admission and treatment initiation. Fetal distress was not evident at that stage. We were therefore able to avoid a caesarean section, and the patient went on to deliver a healthy infant vaginally a few days after discharge from the ICU.

\section{Ethical consideration: Maternal/fetal conflict}

This case also highlights the situation of maternal/fetal conflict that clinicians occasionally need to grapple with when managing antenatal patients. Management for maternal interest necessitates the immediate treatment and stabilisation of the maternal medical condition. However, fetal wellbeing can be compromised in the face of haemodynamic instability, suboptimally resuscitated patients or significant physiological aberrations. In such situations, while the fetus is compromised, immediate delivery is unsafe for the mother, as it may further worsen the physiological derangements and expose her to harm. In conflict-based models, maternal rights are considered to conflict with fetal rights, or moral obligations owed to the pregnant woman are considered to conflict with those owed to the fetus. The key question in the context of possible maternal/ fetal conflict is the status of the fetus: i.e. whether a fetus is a patient or not. According to SA law, the fetus has no legal rights. However, clinicians are increasingly acknowledging the concept of the graded moral status of the fetus, which essentially means that the fetus gains more recognition or moral rights as gestational age increases. As such it is recommended that when maternal/fetal conflicts arise, the ethical principles of respect for autonomy, beneficence (doing good), non-maleficence (doing no harm) and social justice be considered in making an informed decision. Maternal autonomy needs to be respected, and in situations where the patient is incapacitated and unable to participate in such discussions, the clinician should enquire whether the patient had previously expressed her sentiments relating to the issue. In the absence of this, the clinician should consult with the patient's surrogate decision-maker so that the management that offers the patient the greatest balance of benefit over risk is implemented.

\section{Conclusions}

It is of paramount importance that when a pregnant patient presents with ketoacidosis the appropriate cause is determined rapidly, as the management strategy is influenced by the underlying aetiology. In particular, this case illustrates the importance of implementation of early therapy in SKA, as it affects maternal and fetal outcomes.

\section{Acknowledgements. None.}

Author contributions. JMC: literature search, data collection and article content (30\%); FP: literature search, article content (50\%); PS-P, SM and SN: article content and data.

\section{Funding. None.}

Conflicts of interest. None.

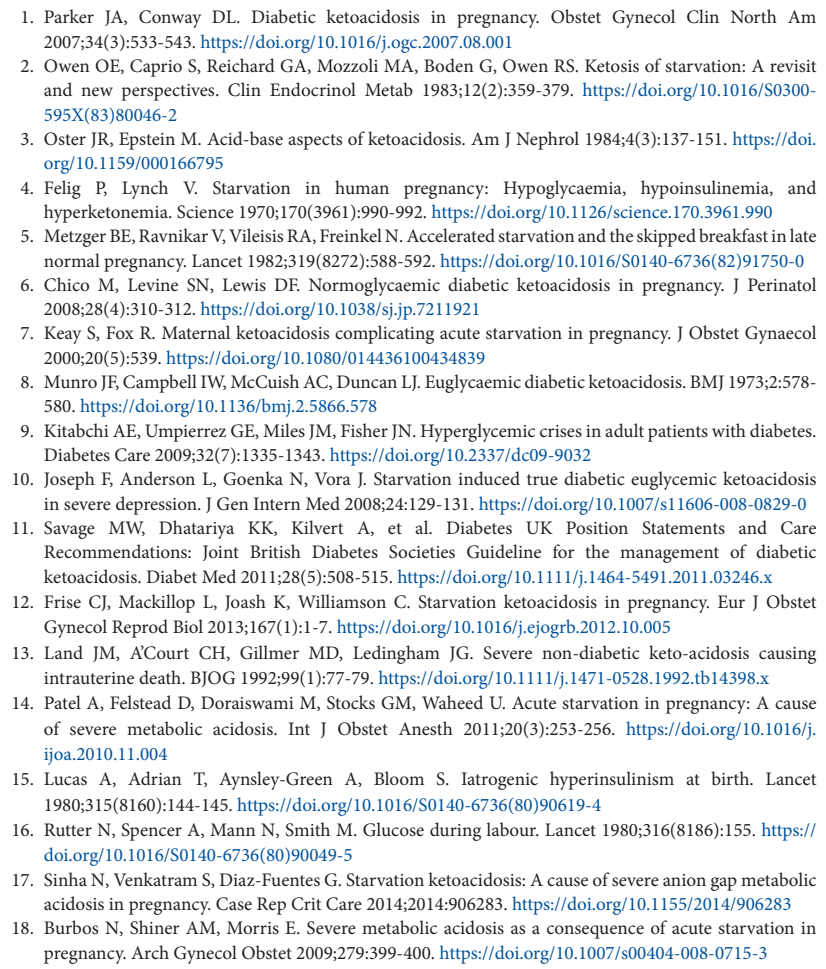
and new perspectives. Clin Endocrinol Metab 1983;12(2):359-379. https://doi.org/10.1016/S0300595X(83)80046-2

3. Oster JR, Epstein M. Acid-base aspects of ketoacidosis. Am J Nephrol 1984;4(3):137-151. https://doi. org/10.1159/000166795

4. Felig P, Lynch V. Starvation in human pregnancy: Hypoglycaemia, hypoinsulinemia, and hyperketonemia. Science 1970;170(3961):990-992. https://doi.org/10.1126/science.170.3961.990

5. Metzger BE, Ravnikar V, Vileisis RA, Freinkel N. Accelerated starvation and the skipped breakfast in late normal pregnancy. Lancet 1982;319(8272):588-592. https://doi.org/10.1016/S0140-6736(82)91750-0 6. Chico M, Levine SN, Lewis DF. Normoglycaemic diabetic ketoacidosis in pregnancy. I Perinatol

2008;28(4):310-312. https://doi.org/10.1038/sj.jp.7211921
7. Keay S, Fox R. Maternal ketoacidosis complicating acute starvation in pregnancy. J Obstet Gynaecol Keay S, Fox R. Maternal ketoacidosis complicating acute
2000;20(5):539. https://doi.org/10.1080/014436100434839

8. Munro JF, Campbell IW, McCuish AC, Duncan LJ. Euglycaemic diabetic ketoacidosis. BMJ 1973;2:578580. https://doi.org/10.1136/bmj.2.5866.578
58.

9. Kitabchi AE, Umpierrez GE, Miles JM, Fisher JN. Hyperglycemic crises in adult patients with diabetes. Diabetes Care 2009;32(7):1335-1343. https://doi.org/10.2337/dc09-9032

10. Joseph F, Anderson L, Goenka N, Vora J. Starvation induced true diabetic euglycemic ketoacidosis in severe depression. J Gen Intern Med 2008;24:129-131. https://doi.org/10.1007/s11606-008-0829-0

11. Savage MW, Dhatariya KK, Kilvert A, et al. Diabetes UK Position Statements and Care Recommendations: Joint British Diabetes Societies Guideline for the management of diabetic ketoacidosis. Diabet Med 2011;28(5):508-515. https://doi.org/10.1111/j.1464-5491.2011.03246.x

12. Frise CJ, Mackillop L, Joash K, Williamson C. Starvation ketoacidosis in pregnancy. Eur J Obstet Gynecol Reprod Biol 2013;167(1):1-7. https://doi.org/10.1016/..ejogrb.2012.10.005

13. Land JM, A'Court CH, Gillmer MD, Ledingham JG. Severe non-diabetic keto-acidosis causing intrauterine death. BJOG 1992;99(1):77-79. https:///doi.org/10.1111/j.1471-0528.1992.tb14398.x
ing

14. Patel A, Felstead D, Doraiswami M, Stocks GM, Waheed U. Acute starvation in pregnancy: A cause of severe metabolic acidosis. Int J Obstet Anesth 2011;20(3):253-256. https://doi.org/10.1016/j. ijoa.2010.11.004

15. Lucas A, Adrian T, Aynsley-Green A, Bloom S. Iatrogenic hyperinsulinism at birth. Lancet 1980;315(8160):144-145. https://doi.org/10.1016/S0140-6736(80)90619-4

16. Rutter N, Spencer A, Mann N, Smith M. Glucose during labour. Lancet 1980;316(8186):155. https:// doi.org/10.1016/S0140-6736(80)90049-5

17. Sinha N, Venkatram S, Diaz-Fuentes G. Starvation ketoacidosis: A cause of severe anion gap metabolic acidosis in pregnancy. Case Rep Crit Care 2014;2014:906283. https://doi.org/10.1155/2014/906283

18. Burbos N, Shiner AM, Morris E. Severe metabolic acidosis as a consequence of acute starvation in pregnancy. Arch Gynecol Obstet 2009;279:399-400. https:// $\mathrm{doi}$. org/10.1007/s00404-008-0715-3
put 